\title{
Pharmaceutical Care Issues in Patients with Atrial Fibrillation Receiving Thromboprophylaxsis
}

\author{
Muhammad Junaid Farrukh*, Shairyzah Ahmad Hisham, Zainol Akbar bin Zainal \\ Faculty of Pharmacy, Cyberjaya University College of Medical Sciences, Malaysia \\ *Corresponding author: junaid_farrukh@live.com
}

Received October 15, 2014; Revised November 20, 2014; Accepted December 04, 2014

\begin{abstract}
Thromboprophylaxis is the mainstay therapy for preventing thromboembolic events such as stroke in Atrial Fibrillation patients. Patients with moderate or high risk of stroke should receive warfarin as it has shown to be superior in clinical trials, with increase in relative reduction of stroke and similar incidence of bleeding compared to aspirin alone. A descriptive cross-sectional study was designed to assess common types and frequencies of PCIs in AF patients receiving thromboprohylaxis and use of thromboprophylactic agents in accordance to stroke risk stratification. Retrospective data was collected by reviewing medical record of hundred patients diagnosed with AF receiving thromboprophylaxis admitted in Serdang Hospital between January-December 2012. Patients were chosen using random sampling technique from PIS data base of the hospital with the help of random number table software. Pharmacist Workup of Drug Therapy (PWDT) form by the Ministry of Health, Government of Malaysia used for patient evaluation in all the hospitals of Malaysia was modified according to the research objectives of the study and was used as data collection tool. Out of 100 patients $(n=56 \%)$ were females while $n=44(44 \%)$ were males. The mean age for males was 60.93 years \pm 7.64 and females was $62.84 \pm 13.52$ years. A total of $15 \%$ patients were not treated according to CPG recommendations regarding thromboprophylaxis. The mean CHA2DS2VASc score was $(3.3, \pm 1.1)$ while mean SD HAS-BLED (bleeding risk) score was $(1.37, \pm 0.92)$. A total of 274 pharmaceutical care issues were identified. The most common pharmaceutical care issues were drug-drug interaction $(n=143)$, noncompliance to treatment $(n=31)$, drug-food interaction $(n=16)$, non-compliance to dietary requirements $(n=15)$ and lack of patient counselling $(\mathrm{n}=35)$.The study reported identification of varieties of PCIs in AF patients receiving thromboprophylaxis which supports the need of dedicated clinical pharmacist to work in collaboration with healthcare team and patients.
\end{abstract}

Keywords: thromboprophylaxis, CHA2DS2VASc Score, Stroke

Cite This Article: Muhammad Junaid Farrukh, Shairyzah Ahmad Hisham, and Zainol Akbar bin Zainal, "Pharmaceutical Care Issues in Patients with Atrial Fibrillation Receiving Thromboprophylaxsis." American Journal of Pharmacological Sciences, vol. 2, no. 5B (2014): 12-16. doi: 10.12691/ajps-2-5B-4.

\section{Introduction}

Atrial fibrillation (AF) is the most common sustained cardiac arrhythmia, occurring in $1-2 \%$ of the general population. AF confers a 5-fold risk of stroke and ischemic strokes in association with AF are often fatal. In addition, those patients who survive are left more disabled by their stroke and are more likely to suffer a recurrence than patients with other causes of stroke. In consequence, the risk of death from AF-related stroke is doubled and the cost of care is increased 1.5-fold [1]. According to Centre for Disease Control (CDC), AF is responsible for 15 to 20 percent of ischemic strokes [2].

Table 1. Thromboprohylaxis management guidelines using CHA2DS2 VASc score

\begin{tabular}{|c|c|c|}
\hline Risk Category & CHA2DS2 VASc score & Recommended therapy \\
\hline $\begin{array}{c}\text { One major risk factor } \\
\text { or } \geq 2 \text { non major risk factors }\end{array}$ & $\geq 2$ & Oral anticoagulants \\
\hline One non major risk factor & 1 & Either oral anticoagulant or aspirin 75-325mg daily, oral anticoagulant is preferred \\
\hline No risk factors & 0 & Either aspirin 75-325mg daily, or no therapy. Preferred no antithrombotic therapy \\
\hline
\end{tabular}

The risk of stroke in AF needs to be assessed in each patient to determine the clinical and cost-effectiveness of thromboprophylaxis, with the aim of appropriate use of antithrombotic therapy. To achieve this, stroke risk factors in AF populations need to be identified and stroke risk stratification models have been devised on the basis of these risk factors [3]. Identification of various stroke clinical risk factors has led to the publication of various stroke risk schemes such as CHADS2 and the latest stroke risk scheme for patients with AF can also be expressed as an acronym, CHA2DS2VASc [congestive heart failure, hypertension, age $\geq 75$ (doubled), diabetes, stroke 
(doubled), vascular disease, age 65-74, and sex category (female)] [4]. Approach to thromboprophylaxis in patients with Atrial fibrillation patients according to European Society of Cardiology (2010) is shown (Table 1) [1].

Long-term management of AF is treated with oral antiplatelet or anticoagulants depending on the risk factors. Numerous clinical trials have provided an extensive evidence for the use of antithrombotic therapy in AF. In a meta-analysis the risk reduction with VKA was highly significant and considered as an absolute annual risk reduction in all stroke cases [5]. Treatment with anticoagulants and antiplatelets put the patient at risk of bleeding. Considering this an assessment of bleeding risk should be part of the patient assessment before starting anticoagulation [6]. HAS-BLED score has been considered appropriate to assess bleeding risk in AF patients, whereby a score of $\geq 3$ indicates 'high risk', and some caution and regular review of the patient is required in initiation of antithrombotic therapy, whether with VKA or aspirin [7].

Pharmaceutical care issues demonstrates the importance of pharmacists working in collaboration with other healthcare providers especially the medical doctors in identifying and resolving pharmaceutical care issues to provide optimal care for patients with chronic diseases [8]. Types of pharmaceutical care issues (PCIs) according to local literature and studies are classified as drug supply/ storage, drug administration, therapeutic choice, adverse drug reactions, predisposing patient factors and special requirements [9]. Several pharmaceutical care issues have been reported in many studies but no comprehensive study has been done on PCIs in AF patients receiving thromboprohylaxis in Malaysia. Therefore, this study was conducted to identify the common types and frequencies of PCIs in AF patients receiving thromboprohylaxis and to assess the use of thromboprophylactic agents in accordance to stroke risk stratification. By identifying common PCIs in AF patients receiving thromboprophylaxis, management strategies can be put into practice to help in optimizing the treatment, thus reducing the risk of stroke and bleeding.

\section{Methods}

\subsection{Study Design}

A descriptive cross-sectional study was designed to assess common types and frequencies of PCIs in AF patients receiving thromboprohylaxis and use of thromboprophylactic agents in accordance to stroke risk stratification. Retrospective data was collected by reviewing medical record of the patients diagnosed with AF receiving thromboprophylaxis admitted in Serdang Hospital between January-December 2012 from PIS.

\subsection{Inclusion and Exclusion Criteria}

The patients, aging $\geq 18$ years old diagnosed with AF receiving thromboprophylaxis were included in the study while those with incomplete medical records such as incomplete INR values or drug histories, pregnant females receiving thrombo-prophylaxis and patients receiving warfarin for indications other than AF were excluded from the study.

\subsection{Sample Size and Technique}

According to WHO for a descriptive study the sample size is of 100 patient encounters from a single facility. Patients were chosen using random sampling technique from PIS data base of the hospital with the help of random number table software.

\subsection{Data Collection Tool}

Pharmacist Workup of Drug Therapy (PWDT) form by the Ministry of Health, Government of Malaysia used for patient evaluation in all the hospitals of Malaysia was modified according to the research objectives of the study and was used as data collection tool. The data collection tool consisted of seven sections. Section 1 of PWDT consisted of demographic data of patients. Section 2 included medical history and risk stratification scores. Stroke risk was assessed by using CHA2 DS2VASc score [congestive heart failure, hypertension, age $\geq 75$. The pharmaceutical care issues with two consultants in pharmacy department. Section 3 consisted of Lab results. Dosage regiment for the drugs used for thromboprophylaxis was included in section 4. Section 5 included patient's current regimen. Section 6 consisted of PCI. Section 7 summarized types of PCI which were identified by researcher in each patient's data base and then reviewed, confirmed and being discussed to ascertain Bleeding risk was assessed by using HAS BLED score [hypertension, abnormal renal/liver function, stroke, bleeding history or predisposition, labile INR, elderly aged 65, drugs/alcohol concomitantly] [11]. (Doubled), diabetes, stroke (doubled), vascular disease, age 65-74, and sex category (female)] [10].

\subsection{Reliability and Validity of Tool}

Focus group discussion of experts of Research committee Hospital Sedang and pilot testing was conducted for reliability and validation of data collection tool. The Cronbach alpha value was found to be 0.76 .

\subsection{Data Analysis}

Data analysis was performed using the software package of SPSS version 16. Categorical data was expressed as frequency and percentage while Chi-squared test $(p \leq 0.05)$ was used to find association among different variables with pharmaceutical care issues.

\section{Results}

\subsection{Demographics}

Total number of patients included in this study were 100. The prevalence of AF was higher among female patients (56\%) compared to male patients (44\%). The mean age \pm SD for males was $60.93 \pm 7.64$ and females was $62.84 \pm 13.52$ years. The highest percentage of age was among 61-70 years old with the youngest being 30 years and oldest 84 years of age. The majority of patients were Malay $(n=53)$, followed by the Chinese $(39 \%)$ and Indian (7\%). All the patient had co-morbidities. The most common co-morbidities were hypertension $(n=60)$, dyslipidemia $(n=35)$, diabetes mellitus $(n=41)$, heart 
failure $(n=38)$, CAD $(n=26)$ and stroke $(n=9)$. Table 2 shows the types of co-morbidities and its frequency.

Table 2. Frequency of demographics and co-morbidities

\begin{tabular}{|c|c|c|}
\hline \multicolumn{3}{|c|}{ DEMOGRAPHICS } \\
\hline \multicolumn{2}{|c|}{ Variable } & Frequency $(\%) n=100$ \\
\hline \multirow{2}{*}{ AGE } & $\leq 65$ & 59 \\
\hline & $\geq 65$ & 41 \\
\hline \multirow{2}{*}{ Gender } & Male & 44 \\
\hline & Female & 56 \\
\hline \multirow{3}{*}{ Race } & Malay & 53 \\
\hline & Chinese & 39 \\
\hline & Indian & 7 \\
\hline \multicolumn{3}{|c|}{ COMORBIDITIES } \\
\hline \multicolumn{2}{|l|}{ Variable } & Frequency (\%), n=100 \\
\hline \multicolumn{2}{|l|}{ Hypertension } & 27 \\
\hline \multicolumn{2}{|l|}{ Dyslipidemia } & 16 \\
\hline \multicolumn{2}{|l|}{ Diabetes Mellitis } & 18 \\
\hline \multicolumn{2}{|l|}{ Heart Failure } & 17 \\
\hline \multicolumn{2}{|c|}{ Coronary artery disease } & 11 \\
\hline \multicolumn{2}{|l|}{ Stroke } & 4 \\
\hline
\end{tabular}

\section{2. $\mathrm{CHA}_{2} \mathrm{DS}_{2} \mathrm{VAS}_{\mathrm{c}}$ and HAS-BLED Bleeding Risk Stratification Score}

The anticoagulants used in long term management of AF were warfarin $(n=57)$ and dabigatran $(n=1)$. The only antiplatelet used was aspirin $(n=3)$. The mean \pm SD $\mathrm{CHA}_{2} \mathrm{DS}_{2} \mathrm{VAS}_{\mathrm{c}}$ Score of study population is $3.3 \pm 1.1$ and $95 \%$ of the patients were identified at high risk of stroke and $5 \%$ at moderate risk. The mean HAD-BLED score was $1.37 \pm 0.92$. $8 \%$ of patients were at high risk of bleeding, 38\% were moderate and 35\% were at low risk of bleeding (Table 3).

Table 3. C $\mathrm{CHA}_{2} \mathrm{DS}_{2} \mathrm{VAS}_{\mathrm{c}}$ Score and HAS-BLED bleeding risk stratification

\begin{tabular}{|c|c|c|c|}
\hline Parameters Score & Low risk F & Moderate risk F & High risk F \\
\hline $\mathbf{C H A}_{2} \mathbf{D S}_{2}$ VAS & 0 & 5 & 95 \\
\hline HAS-BLED & 35 & 38 & 8 \\
\hline
\end{tabular}

\subsection{Association of Bleeding Incidence with Bleeding Risk Stratification and Adherence to CPG Recommendations in Treatment of AF Patients}

The Association of bleeding incidence with bleeding risk stratification shows that there were higher bleeding incidence among those with moderate to high risk of bleeding (HAS-BLED score $\geq 2$ ) compared to those with no risk and low risk. Warfarin and aspirin combination therapy was prescribed in $24 \%$ AF patients with underlying CAD. It is interesting to note that $12 \%$ of patients who received the concomitant therapy of aspirin and warfarin have no previous history of CAD. Majority of the patients were treated according to CPG recommendations. Although more patients among the high-risk group did not receive treatment according to guidelines (Table 4).
Table 4. Association of bleeding with bleeding risk stratification Fisher's exact test significant at $(p \geq 0.05)$

\begin{tabular}{|c|c|c|c|c|}
\hline \multirow[t]{2}{*}{$\begin{array}{c}\text { HAS-BLED } \\
\text { score }\end{array}$} & \multirow[t]{2}{*}{ Risk category } & \multicolumn{2}{|c|}{$\begin{array}{c}\text { Incidence of } \\
\text { bleeding / } \\
\text { bruises }\end{array}$} & \multirow[t]{2}{*}{ p value } \\
\hline & & Yes & No & \\
\hline $0-1$ & No risk to low risk & 2 & 52 & \multirow{2}{*}{0.243} \\
\hline$\geq 2$ & Moderate to high risk & 5 & 41 & \\
\hline \multirow{2}{*}{$\begin{array}{c}\mathrm{CHA}_{2} \mathrm{DS}_{2} \\
\text { VAS }_{\mathrm{c}} \text { score }\end{array}$} & \multirow{2}{*}{$\begin{array}{c}\text { CPG } \\
\text { recommendation }\end{array}$} & \multicolumn{3}{|c|}{$\begin{array}{c}\text { Compliance to } \\
\text { CPG }\end{array}$} \\
\hline & & Yes & & No \\
\hline 1 & Aspirin or OAC & 4 & & 1 \\
\hline$\geq 2$ & OAC & 81 & & 14 \\
\hline
\end{tabular}

\subsection{Impact of Different Variables on PCIs}

PCI were higher in patients aged $\geq 65$ years $(2.92 \pm 2.04)$ as compared to patients aged $<65$ years $(2.79 \pm 2.13)$. However, the mean difference between patient age group and PCIs was statistically insignificant ( $\mathrm{p} \geq 0.05$ ). There is a weak linear correlation between number of PCI and number of comorbidities and the association was statistically insignificant $(\mathrm{p} \geq 0.05)$ whereas there was moderate correlation between number of PCIs and concomitant medications prescribed (Table 5).

Table 5. Impact of different variables on PCIs ( ${ }^{\mathrm{a}}$ Pearson correlation coefficient, ${ }^{\mathrm{b}} \mathrm{p}$ value, ${ }^{\mathrm{C}}$ Spearman correlation coefficient (r))

\begin{tabular}{|l|c|c|c|}
\hline Variable & Age group & Mean ( \pm SD) & $\begin{array}{c}\text { Mean difference } \\
\text { (CI 95\%) }\end{array}$ \\
\hline \multirow{2}{*}{ No of PCIs } & $<65$ & $2.79( \pm 2.13)$ & -.1302 \\
\cline { 2 - 3 } & $\geq 65$ & $2.92( \pm 2.04)$ & $(-0.975,-0.715)$ \\
\hline Variables & \multicolumn{2}{|c|}{ No. of medicines } & Comorbidities \\
\hline \multirow{2}{*}{ No. of PCIs } & \multicolumn{2}{|c|}{$0.427^{\mathrm{a}}$} & $0.095^{\mathrm{c}}$ \\
$0.000^{\mathrm{b}}$ & $0.346^{\mathrm{b}}$ \\
\hline
\end{tabular}

\subsection{Drug Interactions of Warfarin}

Warfarin had more drug-drug interactions ( $n=147)$ compared to aspirin $(n=45)$. The most common drug-drug interaction of warfarin was with statins $(n=60)$, aspirin $(n=38)$, gliclazide $(n=19)$, proton pump inhibitors $(n=10)$ and others $(n=10)$. On the other hand, aspirin had most drug-drug interactions with warfarin $(n=38)$, spironolactones $(n=4)$ and others $(n=3)$ (Table 6).

Table 6. Drug interactions of warfarin

\begin{tabular}{|l|c|}
\hline \multicolumn{1}{|c|}{ Drug interactions of warfarin } & Frequency (\%) \\
\hline Statins & 60 \\
\hline Aspirin & 38 \\
\hline Gliclazide & 19 \\
\hline Propton pump inhibitors & 8 \\
\hline Allopurinol & 6 \\
\hline Amiodarone & 6 \\
\hline
\end{tabular}

\section{Discussion}

Management of AF patients is aimed at reducing symptoms and at preventing severe complications (stroke and thromboembolism) associated with AF. Antiplatelets and anticoagulants are the mainstay of thromboprophylaxis in AF [1]. The present study reported higher prevalence of AF among female patients ( $\mathrm{n}=56 \%$ ) as compared to male 
patients $(n=44 \%)$. The highest prevalent age for AF was 61-70 years with the youngest being 30 years and oldest 84 years of age. Age cut off point at 65 year is used as it is risk parameter in CHA2DS2VASc $\mathrm{S}$ and HAS-BLED score. This results were similar to a study in which mean age of prevalence of AF for male was $60.2 \pm 10.3$ years and mean age for female was $62.5 \pm 9.1$ years [12].

In this study, the anticoagulants used in long-term management of AF were warfarin and dabigatran. The only antiplatelet used was aspirin. These long term management strategy are consistent with the European Society of Cardiology (2010) recommendations [1]. Benefit of using warfarin and aspirin as thromboprophylaxis has been shown in a meta-analysis where the risk reduction with VKA was $2.7 \%$ while with aspirin, there was an absolute risk reduction of $0.8 \%$ per year for primary prevention trials and $2.5 \%$ per year for secondary prevention by using aspirin [5]. In patients with history of coronary artery disease, dual antiplatelet therapy with aspirin plus clopidogrel is recommended, but in AF patients at moderate to high risk of stroke, oral anticoagulant should also be given. An uninterrupted strategy of oral anticoagulant is preferred [1]. In this study combination therapy of warfarin and aspirin was used in $36 \%$ of patients. Among them 24\% patients had underlying coronary artery disease and $12 \%$ were without history of coronary artery disease leading to over treatment and putting the patients at increased risk of bleeding. The combined use of warfarin plus aspirin for secondary stroke prevention is used primarily in patients with Atrial Fibrillation who have concomitant coronary artery disease, however if patient doesn't have concomitant coronary artery disease or structural heart defect, this combination should be avoided [19]. In this study $12 \%$ of patients were given un-necessary aspirin without underlying concomitant coronary artery disease leading to over treatment from which bleeding occurred in 4 cases. This was in consistent with a study in which the combination of aspirin plus warfarin was associated with a significantly increased annual risk for major bleeding side effects, $3.9 \%$ versus $2.3 \%(P=0.01)$ [20].

Few patients were at high risk of bleeding, most of them were at low risk of bleeding. By using this scheme, patients at high risk of bleeding could have been identified and monitored closely to prevent incidence of bleeding. Stroke risk in AF using oral VKA is closely related to bleeding risk. In this study the frequency of bleeding in no risk to low risk HAS-BLED category was less (2\%) and in moderate-high HAS-BLED it was high (5\%). This results were similar to cohort study which concluded that the incidence of both bleeding and adverse cardiovascular events was higher as HAS-BLED score increased [21]. In this study a total of $15 \%$ patients's treatment was not complying with CPG recommendations regarding thromboprophylaxis. Patients (1\%) with CHA2DS2VASc score of 1 were over treated with warfarin and aspirin and among $14 \%$ with CHA2DS2VASc score of $\geq 2$, only $3 \%$ were undertreated and $11 \%$ were over treated although the difference was statistically not significant $(\mathrm{p}=0.56)$. The result was similar to a systematic review which demonstrated the underuse of thromboprophylaxis therapy in AF patients with an elevated risk of stroke [13]. Majority of the patients were treated according to CPG recommendations thus reducing the risk of stroke. This result was quite similar to a cohort study for the validation of risk stratification schemes in which out of 406 patients $92.6 \%$ of them were at high risk when categorized by CHA2DS2VASc score [10].

It has been shown that in elderly patients the numbers of pharmaceutical care issues are more. A study done by Barbara et al. (2011) showed similar results that in the elderly patients reported average medication numbers ranged from 8 to 13, and average numbers of drug-related problems ranging from 2 to 3 and patients with higher age seemed to have higher numbers of medications and drugrelated problem [14].

There was a moderate correlation between number of pharmaceutical care issues and concomitant medication use, and it is statistically significant $(\mathrm{r}=0.42, \mathrm{p}<0.001)$. This result shows that polypharmacy can lead to increase number of pharmaceutical care issues. This is in concordance to a study in which 347 patients were prescribed polypharmacy; increased number of medications was associated with higher risk for patients with full drug related problems on admission [16].

In this study there was weak correlation between number of pharmaceutical care issues and number of comorbidities and the association was statistically not significant ( $p=0.346, r=0.09)$. This result showed that it is not necessary for a patient to have more PCIs if he has more number of co-morbidities. In contrast, a study done by Manley et al. (2003) showed that the number of medication-related problems in an individual patient increases as the number of comorbid conditions increases. Medication-related problems correlated positively with number of patient comorbidities $(p<0.001)$ [15].

Warfarin is efficacious for the treatment and prevention of thromboembolic disorders. Poor compliance with warfarin is common and a major contributor to poor anticoagulation control. A number of psychosocial issues have been associated with warfarin non-compliance among patients in anticoagulation clinics [17]. In this study $31 \%$ of patients were identified as non-compliant to treatment, which resulted in low international normalization ratio. This was consistent to a study in which adherence to long-term warfarin due to the risks of anticoagulantassociated complications and the burden of monitoring [18].

\section{Conclusion}

The study identified various factors involved in inappropriate $\mathrm{PC}$ of AF patients receiving thromboprophylaxsis including drug-drug interactions, non-compliance, dietary requirement, over prescribing and lack of counseling which highlighted the need of dedicated clinical pharmacist to work in collaboration with healthcare team and patients

\section{References}

[1] John Camm. Paulus Kirchhof, Gregory Y.H. Lip, Ulrich Schotten, Isabelle $\mathrm{C}$, Guidelines for the management of atrial Fibrillation, European Heart Journal (2010) 31, 2369-2429.

[2] Brown TM, et al. Heart Disease and Stroke Statistics-2010 Update: a report from the American Heart Association. Circulation. 2010; 121: e91. 
[3] Hughes M, Lip GY. Stroke and thromboembolism in atrial fibrillation: a systematic review of stroke risk factors, risk stratification schema and cost effectiveness data. Thromb Haemost 2008; 99: 295-304.

[4] Nieuwlaat R, Pisters R, Lane DA, Crijns HJ. Refining clinical risk stratification for predicting stroke and thromboembolism in atrial fibrillation using a novel risk factor-based approach: the Euro Heart Survey on atrial fibrillation.Chest 2010; 137: 263-272.

[5] Hart RG, Pearce LA, Aguilare MI. Meta-analysis: antithrombotic therapy to prevent stroke in patients who have nonvalvular atrial fibrillation. Annals of Intern Med 2007; 146: 85767.

[6] Mant J, Hobbs FD, Fletcher K, Roalfe A, Fitzmaurice D, Lip GY, Murray E. Warfarin versus aspirin for stroke prevention in an elderly community population with atrial fibrillation (the Birmingham Atrial Fibrillation Treatment of the Aged Study, BAFTA): a randomised controlled trial. Lancet 2007; 370: 493503.

[7] Pisters R, Lane DA, Nieuwlaat R, de Vos CB, Crijns HJ, Lip GY. A novel userfriendly score (HAS-BLED) to assess one-year risk of major bleeding in atrial fibrillation patients: The Euro Heart Survey. Chest 2010; March 18; 138(5): 1093-100.

[8] Chua, Li Ching Kok, Benny Efendie and Thomas Paraidathathu5 Pharmaceutical care issues identified by pharmacists in patients with diabetes,hypertension, BMC Health Services Research 2012, 12: 388.

[9] Fauzaih Binti Zamri. Pharmaceutical care issues in the treatment og rheumatoid arthritis in adult patients, Cyberjaya university college of medical sciences, 2011 (unpublished).

[10] Jonas Bjerring Olesen, Gregory Y H Lip, professor, Validation of risk stratification schemes for predicting stroke and thromboembolism in patients with atrial fibrillation. British Medical Journal.2011; 342: d124.

[11] Olesen, Anderson. Bleeding risk in _real world_ Journal of Thrombosis and Haemostasis, 2011 Aug; 9(8): 1460-7.
[12] Guize L, Thomas F, Bean K, Benetos A, Pannier B. prevalence, risk factors and mortality in a large French population. Bull Acad Natl Med. 2007 Apr-May;191(4-5):791-803; discussion 803-5.

[13] Ogilvie IM, Newton N, Welner SA, Cowell W, Lip GY. Underuse of oral anticoagulants in atrial fibrillation: a systematic review. $\mathrm{Am}$ J Med. 2010 Jul; 123(7): 638-645.e4.

[14] Barbara Farrell, WaiSum Szeto, and Salima Shamji, Drug-related problems in the frail elderly. Can Fam Physician. 2011 February; 57(2): 168-169.

[15] Manley HJ, McClaran ML, Overbay DK,.Factors associated with medication-related problems. Am J Kidney Dis. 2003 Feb; 41(2): 386-93

[16] Yvonne Koh, Fatimah Bte Moideen Kutty, and Shu Chuen Li. Drug-related problems in hospitalized patients on polypharmacy Therapeutics and Clinical Risk Management 2005: 1(1) 39-48.

[17] Seliverstov I Practical management approaches to anticoagulation non-compliance, health literacy, and limited English proficiency in the outpatient clinic setting. J Thromb Thrombolysis. 2011. 31(3): 321-5.

[18] Arnsten JH, Gelfand JM, Singer DE. Determinants of compliance with anticoagulation: A case-control study. Am J Med. 1997 Jul; 103(1): 11-7.

[19] Dentali F, Doukertis JD, LIM W, Crowther M. Combined aspirinoral anticoagulant therapy alone among patients at risk for cardiovascular disease. Arch Int Med. 2007; 167: 117-124.

[20] Flaker GC, Gruber M, Connolly SJ, Goldman S, Chaparro S, Vabanian A, Halinen MO, Horrow J, Halperin JJ the SPORTIF Investigations. Am Heart J. 2006; 152: 967-973.

[21] Gallego P, Roldán V, Torregrosa JM, Gálvez J, Valdés M, Vicente V, Marín F, Lip GY. Relation of the HAS-BLED bleeding risk score to major bleeding, Circ Arrhythm Electrophysiol. 2012 Apr; 5(2): 312-8. 